\title{
Characterizing Dark State Kinetics and Single Molecule Fluorescence of FusionRed and FusionRed-MQ at Low Irradiances
}

Srijit Mukherjee, ${ }^{1,2}$ Connor Thomas, ${ }^{1}$ Ryan Wilson, ${ }^{1,3}$ Emma Simmerman ${ }^{4}$, Sheng-Ting Hung ${ }^{5}$ and Ralph Jimenez, ${ }^{1,2}$

${ }^{1}$ JILA, University of Colorado, Boulder and National Institute of Standards and Technology, 440 UCB, Boulder, Colorado 80309, United States

${ }^{2}$ Department of Chemistry, University of Colorado, Boulder, 215 UCB, Boulder, Colorado 80309, United States

${ }^{3}$ Department of Physics, University of Colorado, Boulder, 390 UCB, Boulder, Colorado, 80309, United States

${ }^{4}$ Department of Applied Physics, Stanford University, 348 Via Pueblo Mall, Stanford University, Stanford, CA 94305-4090, United States

${ }^{5}$ Department of Biomedical Engineering and Environmental Sciences, National Tsing Hua University, Hsinchu 300044, Taiwan.

\begin{abstract}
The presence of dark states causes fluorescence intermittency of single molecules due to transitions between "on" and "off" states. Genetically encodable markers such as fluorescent proteins (FPs) exhibit dark states that make several super-resolved single-molecule localization microscopy (SMLM) methods possible. However, studies quantifying the timescales and nature of dark state behavior for commonly used FPs under conditions typical of widefield and total internal reflection fluorescence (TIRF) microscopy remain scarce and pre-date many new SMLM techniques. FusionRed is a relatively bright red FP exhibiting fluorescence intermittency and has thus been identified as a potential candidate for SMLM. We herein characterize the rates for dark-state conversion and the subsequent ground-state recovery of FusionRed and its 2.5-fold brighter descendent FusionRed L175M M42Q (FusionRed-MQ) at low irradiances (1-10 W/cm²), which were previously unexplored experimental conditions. We characterized the kinetics of dark state transitions in these two FPs by using single molecule blinking and ensemble photobleaching experiments bridged with a dark state kinetic model. We find that at low irradiances, the recovery process to the ground state is minimally light-driven and FusionRed-MQ has a 1.3-fold higher ground state recovery time indicating a conformationally restricted dark-state chromophore in comparison to FusionRed. Our studies indicate that the brighter FusionRed-MQ exhibits higher tendency in dark state conversion, thus it is potentially a better candidate for SMLM applications than its progenitor FusionRed.
\end{abstract}




\section{Introduction:}

Fluorescence-based bioimaging and biosensing, such as multi-color microscopy, Förster resonance energy transfer (FRET) and fluorescence lifetime imaging microscopy (FLIM), is the primary non-invasive approach to image biological systems. ${ }^{1-6}$ Due to Abbe's diffraction limit, imaging sub-wavelength biological substructures requires innovations beyond the scope of conventional microscopy. ${ }^{7}$ Probing this "super-resolution" scale has high value to modern biology and has garnered the attention of scientists and engineers from a range of specializations - making it a popular interdisciplinary field of research and development. ${ }^{8-13}$ Techniques like stimulated emission depletion microscopy (STED), photoactivated localization microscopy (PALM), reversible saturable optical fluorescence transition microscopy (RESOLFT), and other single-molecule localization microscopy (SMLM) overcome Abbe's limit through biochemical or optical modulation of single emitters. ${ }^{14-17}$ Most SMLM methods, including single-molecule active control microscopy (SMACM), bleaching/blinking assisted localization microscopy (BALM) and super-resolution optical fluctuation imaging (SOFI), rely on photo-activatable or photo-switchable emitters. ${ }^{16}$ These emitters can be activated and/or converted to a new species upon irradiation, exhibiting either molecular binding/modification or spontaneous stochastic blinking. ${ }^{18-22}$ Techniques that exploit stochastic blinking, such as SOFI, rely on post-processed data analysis algorithms where traditionally acquired fluorescence time-lapse images are analyzed to obtain spatio-temporal correlations for stochastic fluctuations during emission. ${ }^{18}$ Current efforts in this domain focus on application-based analysis of such methods and molecular interpretation of the nature of the blinking process for different emitters. A majority of the literature treats emitters simply as tools, with limited analysis of their dark state kinetics and photophysics. ${ }^{23-25}$ Unwanted artefacts in the determination of spatial locations for these emitters can thus arise, as a major assumption underlying such stochastic methods is that the signals from single fluorophores are additive as long as the fluorescence dynamics of the molecules are independent. ${ }^{25}$

Genetically encoded fluorophores, such as fluorescent proteins (FPs), are popular and powerful choices to image biological systems and often exhibit dark molecular states which manifest in stochastic blinking behavior at the single molecule level. ${ }^{26-30}$ Extensive mechanistic studies have elucidated a diverse range of mechanisms and timescales for stochastic and tuned dark-state conversion, including electron-transfer reactions, excited-state proton transfer, chromophore and sidechain conformational changes. For example, a number of proteins from the avGFP line display a spontaneous, light-driven, $\mathrm{pH}$-dependent dark-state conversion pathway. ${ }^{31-33}$ In such cases a $\mathrm{pH}$-dependent dark state conversion is often attributed to a proton transfer between amino acid residues and the chromophore. The red FP (RFP) DsRed exhibits a similar light-driven dark-state conversion through a different, $\mathrm{pH}$-independent process. ${ }^{32}$ Photoconvertible FPs (PCFPs) like IrisFP, mEosFP and Dendra derivatives exhibit dark-state conversion via chromophore distortions due to side-chain conformational changes, chromophore twisting motions or by proton transfer from the triplet or a radical ground state. ${ }^{34-38}$ Selectively engineered photo-switchable or reversiblyswitchable fluorescent proteins (rsFPs) exhibit highly efficient transfer to the dark states, allowing the molecules to be switched on and off with either light-driven $\mathrm{pH}$-dependent pathways or reversible chromophore conformational changes such as a cis-trans isomerization along the methylidine bridge connecting the two conjugated rings in the chromophore moiety. ${ }^{39-41}$ Dark states can be selectively accessed or depopulated using optical or thermal modulation. ${ }^{42-48}$ For example, pulsed excitation with resonant frequencies was used to populate and depopulate the dark state in FPs like AcGFP and rsFastLime, 
providing selective modulation of fluorescence. ${ }^{45}$ Varied mechanisms of accessing dark states as listed above have made FPs natural choices for several SMLM techniques.

Despite the extensive advances in the study of dark-state dynamics, many of the above-mentioned studies far preceded the development of advanced SMLM techniques such as SOFI. Several previous studies were constrained by experimental challenges to probing dark state dynamics with irradiances on the order of $\mathrm{kW} / \mathrm{cm}^{2}$ to attain adequate signal to noise ratios. ${ }^{26-32}$ While these studies explore the fundamentals of dark states in FPs, probing dark-state dynamics at high irradiances comes with a three-fold disadvantage. These conditions accelerate permanent photodegradation of the fluorophore, make extrapolation to much lower irradiance as in widefield and SMLM regimes difficult, and cause light-induced photo-toxicity and photodamage to biological systems. ${ }^{18,49,50}$ It is also worth noting that blue shifting the excitation light increases phototoxicity, thus highlighting the need for development of new bright and red-shifted fluorophores as well as for detailed photophysical characterization of existing red fluorophores - which additionally allow for deeper imaging in comparison to blue shifted analogues. ${ }^{50,51}$ When extrapolating the rate constant of ground-state recovery $\left(\mathrm{k}_{\mathrm{GSR}}\right)$ at low irradiances from high irradiance measurements, $\mathrm{k}_{\mathrm{GSR}}$ is commonly assumed to be proportional to the excitation rate $\left(\mathrm{k}_{\mathrm{Ex}}\right) \cdot{ }^{28,52}$ Recovery from a dark to a fluorescent state is often a consequence of conformational switching such as a dark-trans to fluorescent-cis isomerization of the FP chromophore. ${ }^{53}$ Since such conformational switches are often energetically controlled, the excitation dependence of $\mathrm{k}_{\mathrm{GSR}}$ may originate from the absorption of the excitation photons by dark state species and/or the rise of local temperature due to high irradiance. ${ }^{28,52,54}$

FusionRed and its sibling TagRFP-T exhibit fluorescence intermittency in live-cell imaging using TIRF microscopy with camera acquisition timescales of $50 \mathrm{~ms} .{ }^{55}$ The study demonstrated the potential to achieve a theoretical spatial resolution beyond the diffraction limit $(\sim 25-30 \mathrm{~nm})$ with FusionRed using SMLM methods like BALM and SOFI. ${ }^{55}$ In our previous work, we developed FusionRed-MQV, a FusionRed variant with 3-fold higher molecular brightness developed using a combination of lifetime-based microfluidic selection and site-directed mutagenesis. ${ }^{53}$ Additionally, we found that the substitution C159V in FusionRed resulted in a brighter variant which showed a monoexponential photobleaching trace contrary to the biexponential behavior exhibited by the parental RFP FusionRed. Variants lacking the C159V substitution, like the 2.5-fold brighter FusionRed-MQ exhibited biexponential photobleaching traces with a fast fluorescence decay component $(\sim \mathrm{s})$ followed by a significantly slower decay component $(>100 \mathrm{~s}) .{ }^{53}$ The faster component was attributed to reversible photobleaching, where fluorescent molecules are trapped in the dark state..$^{53}$ This was verified by employing a high-energy $438 \mathrm{~nm}$ pulse ( $2 \mathrm{~s} ; 50 \%$ duty) with a continuous $560 \mathrm{~nm}$ excitation scheme which resulted in distinct reversible photoswitching for FusionRed variants with a Cys residue at position $159 .{ }^{53}$ High energy $438 \mathrm{~nm}$ light prompts a return to the fluorescent state from a dark state, suggesting that the lower energy $560 \mathrm{~nm}$ excitation minimally perturbs the ground state recovery process. ${ }^{53}$ Together with findings and from other studies, including crystal structure data, indicate a possible interconversion of the FusionRed chromophore from a fluorescent cis to a dark trans isomer ${ }^{56,57}$ Moreover, in the low irradiance regime of $1-10 \mathrm{~W} / \mathrm{cm}^{2}$, the temperature increase in the vicinity of an FP molecule can be considered negligible. ${ }^{58}$ Based on these observations, we hypothesized that the rate of recovery to the ground state $\left(\mathrm{k}_{\mathrm{GSR}}\right)$ is independent of the excitation rate $\left(\mathrm{k}_{\mathrm{Ex}}\right)$ under low irradiances for FusionRed and FusionRed-MQ. To verify these claims, we explore the relatively uncharted territory of dark state kinetics of these two RFPs under low irradiances, central to widefield and SMLM techniques like SOFI. We do so by extracting on-off statistics with single molecule imaging and exploiting the kinetics of reversible photobleaching using widefield excitation on ensemble RFPs. We combined the strength of each 
approach to quantitatively extract rate constants of dark-state conversion $\left(\mathrm{k}_{\mathrm{DSC}}\right)$ and ground-state recovery ( $\mathrm{k}_{\mathrm{GSR}}$ ) using a three-state model (Figure 1). We performed simulations utilizing Monte Carlo methods to bridge the two extremes in imaging modalities and qualitatively validate the three-state model. Finally, we propose a structural model to rationalize the dark-state dynamics observed for these FPs.

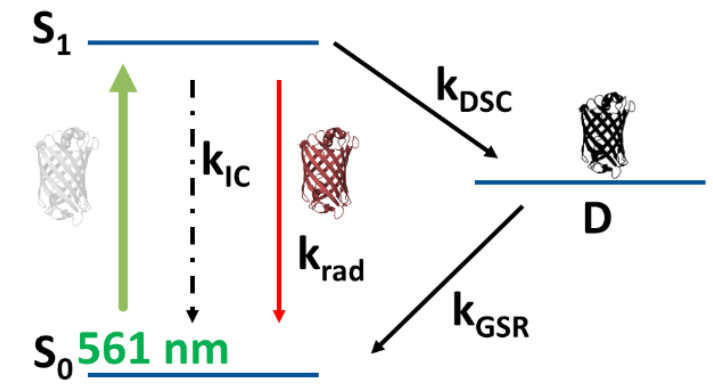

Figure 1: A three-state model of the photophysics of a fluorophore central to this study. The $561 \mathrm{~nm}$ excitation source allows access to the excited electronic $\left(\mathrm{S}_{1}\right)$ state from the ground electronic $\left(\mathrm{S}_{0}\right)$ state. Following this, the fluorophore can either return to $\mathrm{S}_{0}$ or access a long lived dark (D) state. It was demonstrated in our previous work that the D state can be depopulated efficiently using a $438 \mathrm{~nm}$ light. ${ }^{53}$ The arrow labels $\mathrm{k}_{\mathrm{ex}}, \mathrm{k}_{\mathrm{IC}}, \mathrm{k}_{\mathrm{rad}}, \mathrm{k}_{\mathrm{DSC}}$ and $\mathrm{k}_{\mathrm{GSR}}$ indicate the rate constants for excitation, non-radiative (internal conversion), radiative emission, $S_{1}$ to dark state conversion and dark state to $S_{0}$ recovery, respectively. Permanent photobleaching from the $S_{1}$ and the D states are ignored in the regime of low irradiances.

\section{Methods}

\section{(a) Experimental methods and data collection}

\section{Cell growth and protein purification:}

FusionRed and FusionRed-MQ in the pBad-His plasmid were transformed into the E. coli Top10 strain via heat shock and grown for 45-60 minutes in LB media in a shaker at $37{ }^{\circ} \mathrm{C}$ and $230 \mathrm{rpm} .{ }^{53}$ The transformants were plated on an agar plates with $100 \mu \mathrm{g} / \mathrm{mL}$ ampicillin and $0.2 \%$ arabinose (Sigma Aldrich) overnight at $37^{\circ} \mathrm{C}$. Colored colonies were grown in $200 \mathrm{~mL}$ 2XYT (VWR) liquid cultures with $100 \mu \mathrm{g} / \mathrm{mL}$ ampicillin for $1-3$ hours at $37{ }^{\circ} \mathrm{C}$ and $230 \mathrm{rpm}$ to an OD of 0.6. Arabinose was then added $(0.2 \%)$ to induce protein expression for $16-24$ hours at $28{ }^{\circ} \mathrm{C}$ and $230 \mathrm{rpm}$. The cells were pelleted, chemically lysed (B-PER, Thermo Fisher Scientific) and the 6-His tagged FPs were isolated on Ni-NTA columns (Thermo Fisher Scientific) by gravity filtration, eluting with $250 \mathrm{mM}$ imidazole (Sigma Aldrich). Excess imidazole was removed with desalting columns (GE Healthcare) with dialysis buffer (150 mM NaCl, $50 \mathrm{mM}$ Tris-HCL, $\mathrm{pH}$ 7.4) as an eluent.

\section{Single molecule measurements:}

Preparation of glass slides and coverslips: Minimizing the presence of fluorescent impurities is of particular concern in single-molecule studies. To reduce artefacts from impurities in our measurements, we found that plasma-cleaned glass slide chambers were best suited to single molecule TIRF. ${ }^{59}$ Before plasma cleaning, the slides and coverslips ( $22 \times 40 \mathrm{~mm}$, No 1, VWR) were cleaned with dilute $\mathrm{HCl}$ then washed with Alconox detergent and rinsed with deionized water, then soaked in methanol overnight to dislodge 
large contaminants. A custom aluminum slide holder held the slides and coverslips inside a reactive ion etcher, such that both sides were exposed to the plasma. The slides were then exposed to $300 \mathrm{~s}$ of $\mathrm{O}_{2}$ (Bias: $50 \mathrm{~W}$ and SCCM: 50 mTorr) plasma to remove organic contaminants and to charge their surfaces, followed by 60 s of Ar plasma (Bias: $50 \mathrm{~W}$ and SCCM: $50 \mathrm{mTorr}$ ) to minimize presence of remaining reactive oxygen species. The slides were used within 24 hours after plasma cleaning to avoid recontamination and loss of surface charge.

Sample preparation: The pure protein samples were diluted with Tris-HCl buffer ( $\mathrm{pH} ~ 7.4-8.0$ ) and loaded by slow ejection from a $200 \mu \mathrm{L}$ micropipette. It was determined that FP concentrations $>300 \mathrm{pM}$ caused crowding of FPs in the field of view and failure of our spot analysis algorithm to report blinking trajectories, whereas concentrations $<100 \mathrm{pM}$ resulted in such sparse distribution that it became difficult to find the correct focus height and provided few data points. Additionally, a washing procedure was developed to minimize the presence of non-adhered FPs in solution and thus minimize free FP diffusion into the imaging plane. The loaded chamber was left in the dark for 10-15 minutes to allow FPs to settle onto the imaging surface, then a volume of imaging buffer (150 mM HEPES, $100 \mathrm{mM} \mathrm{NaCl}, \mathrm{pH} 7.4$ ) equal to the volume of the loaded sample was passed through the chamber 4-6 times, with 2-minute intervals between washes. The liquid was slowly ejected by a micropipette on one side of the chamber while filter paper was used to absorb the liquid flowing out from the other side. This washing procedure helped to maximize signal-tobackground ratios and minimize artifacts from FPs in solution.

TIRF Imaging: The samples were imaged with TIRF microscopy on an Olympus IX-73 inverted microscope. The microscope is accessorized with an Olympus cellTIRF-1Line system fiber coupled to a laser (Toptica iChrome MLE). An Olympus 60x-in-oil (NA:1.42) TIRF objective and an EMCCD camera (Andor iXon 897) were used for the single molecule experiments. A schematic of this system has been provided in Supplementary Information S1; Figure S1.1. To measure the excitation rate, the objective focus was first determined by imaging a dye sample under bright-field illumination, then the sample was removed and the laser was focused at the ceiling (approximately $2 \mathrm{~m}$ beyond the sample location) for this z-position of the objective. The irradiance measurements were carried in this normal $\left(\mathrm{I}_{\text {Normal }}\right)$ to the imaging plane position using a power meter (X-cite). The excitation intensity of the evanescent field ( $\mathrm{I}_{\text {TIRF }}$ ) was calculated from the incident intensity ( $\left.\mathrm{I}_{\text {Normal }}\right)$, the indices of refraction $\left(\eta_{2}, \eta_{1}\right)$, and the incident angle. ${ }^{60}$ The calculations of excitation rates for normal and TIRF illumination are presented in Supplementary Information S2. To image samples, a cropped area of the imaging plane ( 128 x 128 pixels on a $256 \times 256-$ pixel binning) corresponding to the region of highest intensity of the laser profile was selected. Then for the lowest value of irradiance $\left(1 \mathrm{~W} / \mathrm{cm}^{2}\right), 100 \mathrm{~nm}$ fluorescent beads (TetraSpeck) were used to first determine the approximate $\mathrm{z}$-focus, and the motorized stage (Prior) was moved in the $\mathrm{x}-\mathrm{y}$ plane to the position of single FP molecules, to determine an accurate focus. The experiment was started after moving to an adjacent spot $(\sim 100 \mu \mathrm{m})$ outside this imaging area of the previous step, where drift on the z-axis was minimal. This was done so as to minimize photobleaching of single molecules.

\section{Ensemble measurements:}

Bright bacterial colonies on the agar plates described above were chosen for time-lapse photobleaching experiments. Two to three colonies were transferred to microcentrifuge tubes and washed with $500 \mu \mathrm{L}$ imaging buffer by vortexing for $20 \mathrm{~s}$. The cells were centrifuged at 3000-5000 RPM for $60 \mathrm{~s}$, and the washing buffer was removed. The pelleted cells were then resuspended in the same buffer to an OD in the 
range of 0.1 to 0.5 to get a cell density suitable for imaging. A glass coverslip and slide were cleaned with Alconox detergent, rinsed with deionized water, and blown dry with filtered compressed air. 10-20 $\mu \mathrm{L}$ of the cell mixture was added between the coverslip and slide, which was imaged on an Olympus IX-73 inverted microscope system. Samples were excited by $560 \mathrm{~nm}$ continuous wave LED illumination (Lumencor). Fluorescence bleaching measurements were taken with the 20x or 40x-in air objective lens (Olympus). The fluorescence was collected through a $629 / 56 \mathrm{~nm}$ band-pass filter by a SCMOS camera (Andor Zyla). Videos were collected with 10-50 ms exposure times and frame rates of 20-32 FPS for the fast and reversible component of the decay and 10-20 FPS for the slow and irreversible component, and with irradiances ranging from $1-20 \mathrm{~W} / \mathrm{cm}^{2}$. We performed three independent trials where each trial for an FP involved a technical replicate with $\sim 10-20$ cells to gain consistent bleaching traces.

\section{(b) Data analysis}

\section{Single molecule data analysis:}

Single molecule data analyses from imaging videos were carried out using two independent scripts: One for spatial identification of bright spots followed by one for temporal and intensity analysis of these bright spots. Figure 2 shows a schematic representation of this workflow.

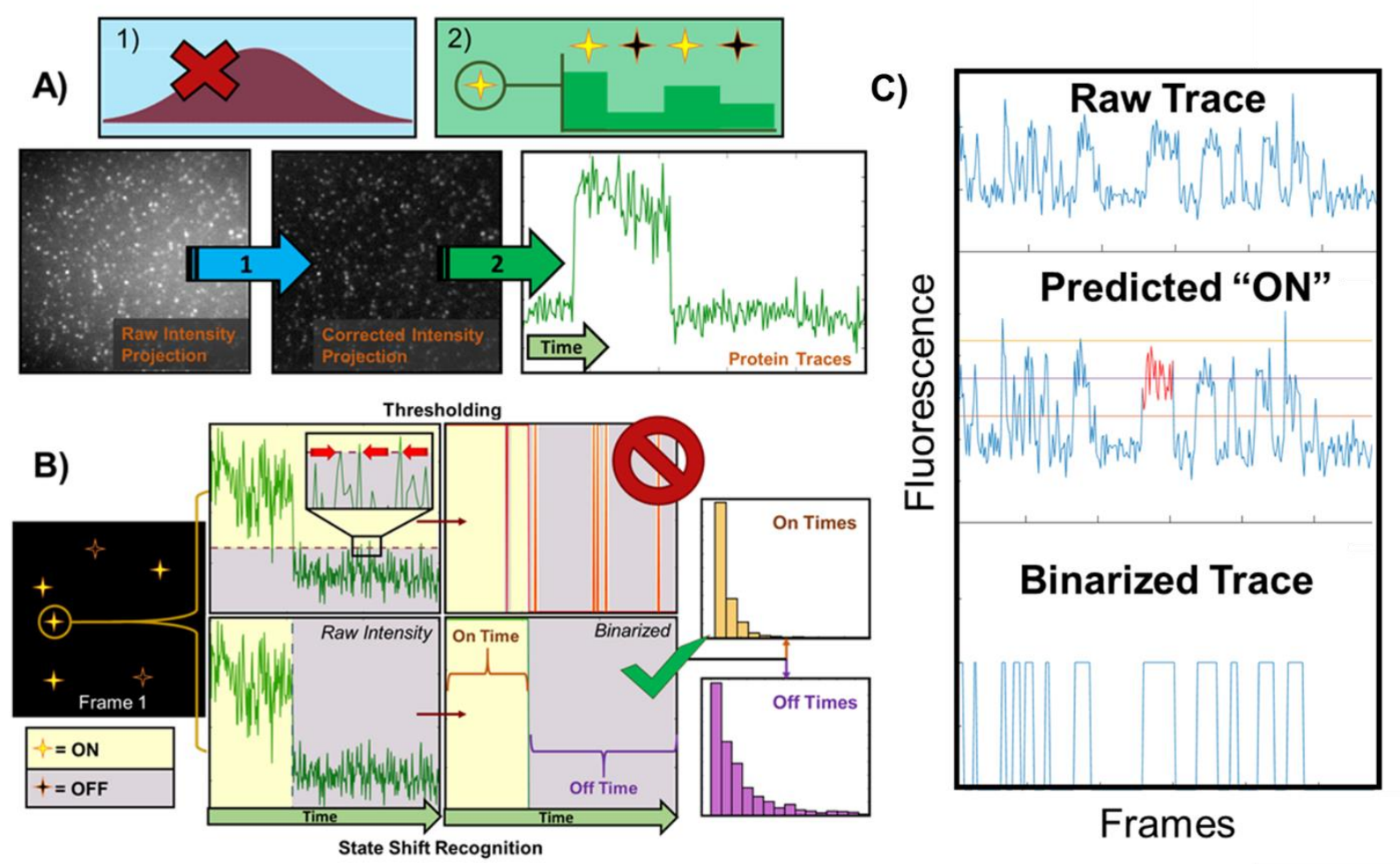

Figure 2: Analysis workflow. A) The background correction and molecule selection process. B) Our analysis workflow contrasted against standard thresholding methods. Note that the state shift recognition workflow identifies points where possible changes occur and binarizes on the basis of those points. This avoids the noise-based artifacts shown in the thresholding approach, where it can be difficult to set an arbitrary threshold that is not crossed by noise. C) The binarization of a raw trace based on the thresholding algorithm. 
Spot identification script: Despite cropping, there is a systematic $\sim 10 \%$ intensity variation across the imaging plane with a Gaussian profile. To account for this, the videos were iteratively fitted to a Gaussian intensity correction function to correct for the laser background, primarily from residual scattering, and normalizing for the variation in intensity due to the spatial mode of the excitation laser. (Supplementary Section S3; Figure S3.1) Following Gaussian correction, our analysis also revealed a biexponential decay of mean intensities across the timeframe of the video. Therefore, the mean intensity of the videos was fitted to a biexponential function, which revealed a fast component of decay $(\sim 1 \mathrm{~s})$ along with a slow component of decay (>3 s). While the timescale of the slow component of decay varied with the incident intensity, the fast component was seen to be fairly consistent (Supplementary Information S3; Figure S3.1c). Additional checks with blank solutions also revealed a consistent timescale for the fast component of decay. Therefore, after the Gaussian correction, a secondary correction was incorporated to account for the quick exponential drop in the overall light intensity. Given that this decay was also found in blank medium and was missing from the laser's temporal profile, we attribute it to diffusion or photobleaching of impurities in our blank medium or the objective oil. Following the Gaussian and exponential corrections, the algorithm identifies a number of bright locations equal to an input of the predicted number of single FPs in the video. This number was set between 50 and 500 FPs depending on the field of view, the efficiency of binding the FPs to the glass surface and the concentration of the protein used. The algorithm extracts the brightest pixels in the maximum intensity projected image of the video from the user defined input value for the number of single FPs. It then iteratively appends the location of maximum value after it passes a check, which involves scanning a pixel grid surrounding the pixel centered at maximum value based on the statistical distribution of the brightness around the grid.

Temporal and Intensity analysis: To extract information on real "on" and "off" blinking events we drew inspiration from the work of Watkins and Yang. ${ }^{61}$ In order to find single on and off events in a trajectory of a single molecule, we used an intensity change point approach. Our spot analysis script provides us with intensity corrected trajectories with time for the brightest spots arranged in the ascending order of mean intensity. We therefore assessed the first five and the last five trajectories, based on the brightest and the dimmest spots identified through the previous script. In each case, we estimated the average single molecule on intensity to provide as an input for this code. Although many approaches utilize histograms from the intensities of each frame to effectively threshold and binarize a trajectory, it is difficult to use this approach for our data sets at the lower and the upper bounds of irradiances, which are characterized by increasingly longer $\tau_{\mathrm{ON}}$ or $\tau_{\mathrm{OFF}}$, respectively. ${ }^{62}$

Following the input of the five potential on events, our algorithm performs two steps. First, it fits the change of intensity between frames for the entire dataset to a Gaussian distribution. It should be noted that a protein turning on or off produces a relatively small change in intensity that falls within the noise distribution. Therefore, it is not possible to separate these events from background noise with equal or higher intensity without additional information, whereas noise below this threshold can be discarded. As a result, all frames with a change value of the threshold or higher are earmarked as possible changes of state. Thus, the primary question is where to set the threshold for optimal recognition. (Supplementary Information S3b; Figure S3.2) The theoretical minimum intensity changes for a molecule results from a case where the protein turns on exactly halfway through a frame's acquisition time. This results in a change of intensity of $1 / 2$ the protein signal, followed by a second change of the same magnitude. As such, a good baseline estimate for the noise threshold value is $50 \%$ of the expected signal. Empirically, we have found that $\sim 1.5 \sigma$ (standard deviation) 
of the overall noise produced consistent results. This boundary excludes $86 \%$ of overall noise, but it is worth noting that the on state contributes to higher (shot) noise. As such, this boundary excludes approximately $70 \%$ of the larger standard deviation on distribution. The second step of the algorithm considers the intervals between each on and off point individually and binarizes each segment on the basis of a user-defined $\tau_{\mathrm{ON}}$. (Supplementary Figure S3.2 and Figure 2C) This further minimizes the contributions of noise by averaging each segment. Segments that are more than $2 \sigma$ above the model $\tau_{\mathrm{ON}}$ are considered multi-molecule events and the corresponding trace is discarded. All segments that end at the last frame of the video (on or off) are also discarded to avoid artifacts, notably from permanent photobleaching.

\section{Ensemble data analysis:}

Photobleaching data analysis was carried out with a previously reported scheme. ${ }^{53}$ In brief, a pipeline in the CellProfiler suite ${ }^{63}$ was used to identify bacteria in an imaging plane of uniform irradiance and provide normalized intensity trajectories. The intensity trajectories were analyzed and fit to biexponential traces using a custom fitting program in MATLAB (Supplementary Section S4; Table S4.1).

\section{Results:}

(a) Simulations and measurements of single molecule photophysics: We performed simulations of blinking trajectories for single molecules in a three-state $\left(S_{0}, S_{1}\right.$ and $\left.D\right)$ model with our hypothesis. (Figure 1) As expected, the results show that $\tau_{\mathrm{ON}}$ decreases with both shorter values of $\tau_{\mathrm{DSC}}$ and higher values of $\mathrm{k}_{\mathrm{Ex}}$, whereas $\tau_{\mathrm{OFF}}$ depends only on the value of $\tau_{\mathrm{GSR}}$. The details of the algorithm and simulated results are described in the Supplementary Information Section S5. In brief, the residence of single FPs in each state $\left(\mathrm{S}_{0}, \mathrm{~S}_{1}\right.$ or $\left.\mathrm{D}\right)$ at each time step is simulated using a combination of Monte Carlo methods weighted by probabilities of state change, governed by the $\mathrm{k}_{\mathrm{Ex}}, \mathrm{k}_{\mathrm{rad}}, \mathrm{k}_{\mathrm{non}-\mathrm{rad}(\mathrm{IC})}, \mathrm{k}_{\mathrm{DSC}}$ and $\mathrm{k}_{\mathrm{GSR}}$. An "on" or a fluorescent event is detected every time the molecule successfully completes an $\mathrm{S}_{0}-\mathrm{S}_{1}-\mathrm{S}_{0}$ cycle, without going through D. (Figure 1) The predictions of the simulations accurately represent experiments, where data from three independent trials revealed a hyperbolic dependence of the $\tau_{\mathrm{ON}}$ of FusionRed and FusionRed-MQ on the excitation rates. The $\tau_{\mathrm{OFF}}$ for both FPs showed little or no dependence on excitation rates as summarized in Table 1 and Figure 3.

Our single molecule measurements were carried out with irradiation on the order of $\sim 10^{3}$ photons/s, which is significantly lower than the optical saturation limit for FPs $\left(\sim 10^{8}\right.$ excitation-photons/s). Therefore, the population in the $S_{1}$ electronic state can be treated under a steady state approximation. At low irradiances, we assume minimal absorption from the dark states and therefore consider the reverse dark-state conversion to be negligible. ${ }^{53}$ The timescales of permanent photobleaching are significantly longer than that of darkstate lifetime (Supplementary Section S4), hence it was neglected in this scheme. Incorporating these assumptions, we arrive at equations 1 and 2 to calculate $\mathrm{k}_{\mathrm{DSC}}$ and $\mathrm{k}_{\mathrm{GSR}}$ for FusionRed and FusionRed-MQ, ${ }^{64}$ and the calculated values are presented in Table 2 and Figure 4.

$$
\begin{gathered}
\tau_{O N}=\frac{k_{e m}+k_{n o n-r a d}+k_{d s c}}{k_{e x} \cdot k_{d s c}} \\
\tau_{O F F}=\frac{1}{k_{G S R}}(2)
\end{gathered}
$$


Table 1: Values of $\tau_{\mathrm{ON}}$ and $\tau_{\mathrm{OFF}}$

\begin{tabular}{|c|c|c|c|c|c|}
\hline \multicolumn{3}{|c|}{ FusionRed } & \multicolumn{3}{c|}{ FusionRed-MQ } \\
\hline $\mathbf{K E x}_{\mathbf{E x}}\left(\mathbf{s}^{\mathbf{- 1}}\right)$ & $\boldsymbol{\tau}$ On $(\mathbf{s})$ & $\boldsymbol{\tau}$ Off $(\mathbf{s})$ & kEx $_{\left(\mathbf{s}^{\mathbf{1}}\right)}$ & $\boldsymbol{\tau}$ On $(\mathbf{s})$ & $\boldsymbol{\tau}$ Off $(\mathbf{s})$ \\
\hline 950 & $10.9 \pm 2.3$ & $5.3 \pm 0.5$ & 1600 & $4.9 \pm 0.3$ & $6.4 \pm 1.5$ \\
\hline 1200 & $9.5 \pm 1.1$ & $4.7 \pm 1$ & 2400 & $3.5 \pm 1.1$ & $6.6 \pm 0.5$ \\
\hline 1400 & $6.2 \pm 0.9$ & $5.2 \pm 0.2$ & 3300 & $2.8 \pm 0.3$ & $6.1 \pm 0.1$ \\
\hline 1900 & $4.4 \pm 0.4$ & $4.8 \pm 0.7$ & 5200 & $2.0 \pm 0.3$ & $6.5 \pm 0.5$ \\
\hline 6000 & $2.6 \pm 0.5$ & $5.3 \pm 1.2$ & 10400 & $1.3 \pm 0.4$ & $6.5 \pm 1.0$ \\
\hline 12000 & $1.2 \pm 0.5$ & $5.8 \pm 0.8$ & & & \\
\hline
\end{tabular}

A)

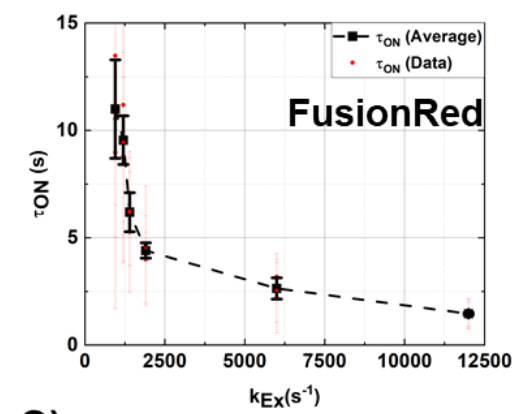

$$
\text { C) }
$$

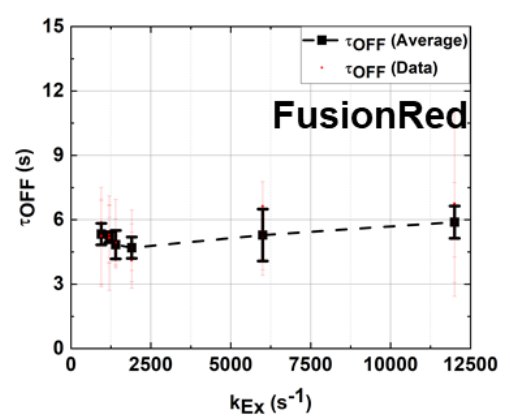

B)

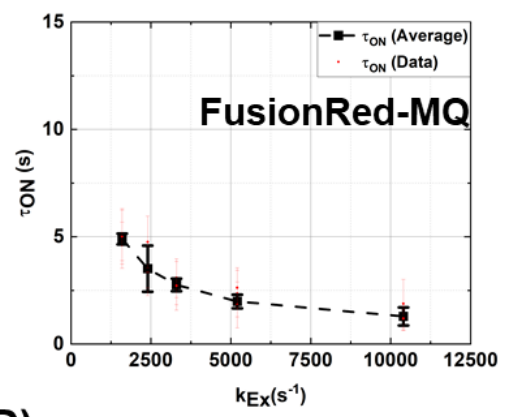

D)

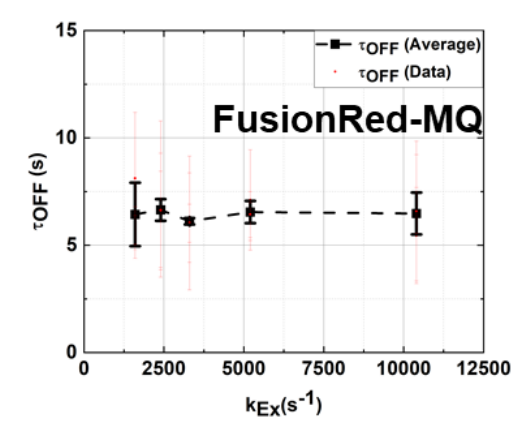

Figure 3: Results from single molecule blinking experiments: Measured $\tau_{\mathrm{ON}}$ with respect to excitation rate for $\mathrm{A}$ ) FusionRed and B) FusionRed-MQ; and measured $\tau_{\mathrm{ON}}$ with respect to excitation rate for C) FusionRed and D) FusionRed-MQ. The black data points are the mean $\tau_{\mathrm{ON} / \mathrm{OFF}}$ extracted from three independent experiments (error bars indicate the confidence intervals for the values extracted from fits). 
Table 2: Estimation of $k_{D S C}$ and $k_{G S R}$

\begin{tabular}{|c|c|c|c|c|c|}
\hline \multicolumn{3}{|c|}{ FusionRed } & \multicolumn{3}{c|}{ FusionRed-MQ } \\
\hline $\mathbf{k}_{\mathbf{E x}}\left(\mathbf{s}^{\mathbf{- 1}}\right)$ & $\mathbf{k}_{\mathbf{D S C}}\left(\mathbf{x 1 0 ^ { 3 }} \mathbf{s}^{\mathbf{- 1}}\right)$ & $\mathbf{k}_{\mathbf{G S R}}\left(\mathbf{s}^{\mathbf{- 1}}\right)$ & $\mathbf{k}_{\text {Ex }}\left(\mathbf{s}^{\mathbf{- 1}}\right)$ & $\mathbf{k}_{\mathbf{D S C}}\left(\mathbf{x 1 0}^{\mathbf{3}} \mathbf{s}^{\mathbf{- 1}}\right)$ & $\mathbf{k}_{\mathbf{G S R}}\left(\mathbf{s}^{\mathbf{- 1}}\right)$ \\
\hline 950 & $55 \pm 11$ & $0.19 \pm 0.02$ & 1600 & $52 \pm 4$ & $0.16 \pm 0.02$ \\
\hline 1200 & $50 \pm 5$ & $0.21 \pm 0.03$ & 2400 & $48 \pm 15$ & $0.15 \pm 0.01$ \\
\hline 1400 & $60 \pm 7$ & $0.19 \pm 0.01$ & 3300 & $46 \pm 5$ & $0.16 \pm 0.01$ \\
\hline 1900 & $67 \pm 10$ & $0.21 \pm 0.03$ & 5200 & $40 \pm 6$ & $0.15 \pm 0.01$ \\
\hline 6000 & $39 \pm 10$ & $0.19 \pm 0.04$ & 10400 & $36 \pm 10$ & $0.15 \pm 0.02$ \\
\hline 12000 & $39 \pm 16$ & $0.18 \pm 0.03$ & & & \\
\hline
\end{tabular}
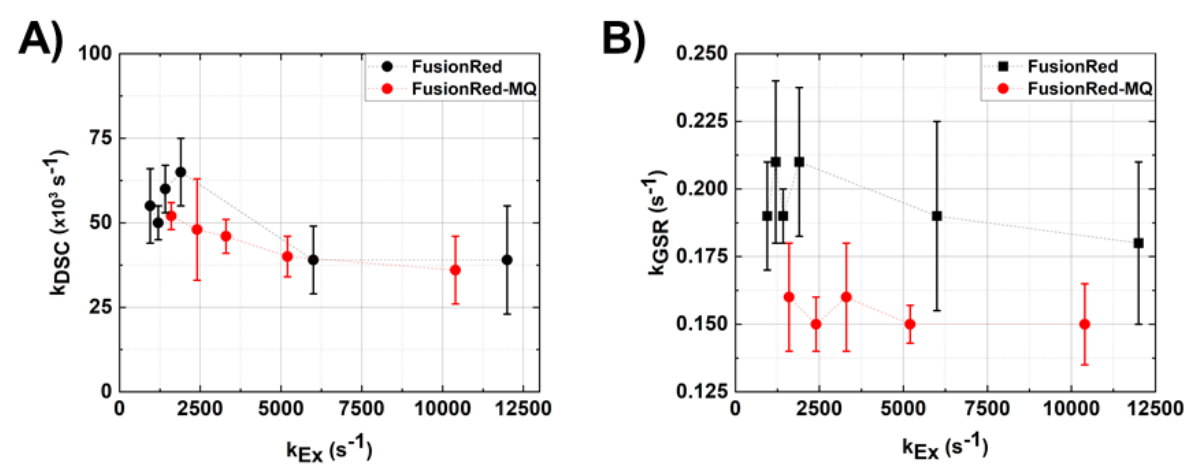

Figure 4: Variation of rate constants A) $\mathbf{k}_{\mathbf{D S C}}$ and B) $\mathbf{k}_{\mathbf{G S R}}$ with respect to excitation rate $\mathbf{k}_{\mathbf{E x}}$ measured from singlemolecule blinking experiments. The error bars indicate standard deviation errors calculated from measured $\tau_{\mathrm{OFF}}$ and $\tau_{\mathrm{ON}}$.

The $\mathrm{k}_{\mathrm{DSC}}$ and $\mathrm{k}_{\mathrm{GSR}}$ remain consistent under the range of irradiances probed in this study. However, FusionRed clearly shows an $\sim 30 \%$ higher $\mathrm{k}_{\mathrm{GSR}}$ than FusionRed-MQ.

(b) Simulating ensemble behavior: We first generated normalized sum fluorescence traces for $\sim 10^{3}-10^{4}$ simulated blinking single FP trajectories using the average $\mathrm{k}_{\mathrm{DSC}}$ and $\mathrm{k}_{\mathrm{GSR}}$ from the single molecule experiments. The simulations indicated a larger dark fraction for FusionRed-MQ in comparison to FusionRed. We then performed simulations to obtain normalized sum fluorescence traces for blinking single FP trajectories ( 3 sets of 150 emitters each) by varying one rate constant at a time, including $\mathrm{k}_{\mathrm{Ex}}$, $\mathrm{k}_{\mathrm{DSC}}$ and $\mathrm{k}_{\mathrm{GSR}}$. The results indicated that the dark fraction increases with increasing $\mathrm{k}_{\mathrm{Ex}}$ and $\mathrm{k}_{\mathrm{DSC}}$ but has no or little dependence on $\mathrm{k}_{\mathrm{GSR}}$ in the vicinity of measured values in the single molecule blinking experiments. This observation encouraged us to pursue an alternate analysis compared to single molecule experiments, to quantify the $\mathrm{k}_{\mathrm{DSC}}$. The results of these simulations are presented in Supplementary Information Section $\mathrm{S} 5$ ( $\mathrm{f}, \mathrm{g}$ and $\mathrm{h}$ ). The normalized fluorescence signals are proportional to the normalized population of FPs on the ground state $S_{0}$, thus the fractions of FPs on $S_{0}$ and dark state $D$ as a function of time can be estimated as shown in Supplementary Figure S5.9.

(c) Ensemble photobleaching: E. coli expressing FusionRed and FusionRed-MQ were exposed to normalized widefield excitation. The decay profiles of both FPs were recorded and fit with a biexponential function as presented in Supplementary Information S4. Based on our previously reported pulsed 
photobleaching measurements, we assigned the slower decay component to permanent photobleaching and the faster component to reversible photobleaching due to dark-state conversion. ${ }^{53}$ Experimental results showed a larger dark fraction for FusionRed-MQ compared to FusionRed.

To extract $\mathrm{k}_{\mathrm{DSC}}$, we consider a three-state model to represent the kinetic processes of a fluorescent protein in the first five seconds of a photobleaching trace, where the contribution of the permanent bleaching component is minimal $(<10 \%)$ and reverse dark-state conversion $\left(\mathrm{D}\right.$ to $\mathrm{S}_{1}$ ) can be ignored. The rate equations can be written in the matrix form, $\frac{d \boldsymbol{n}}{d t}=A \boldsymbol{n}$, as explicitly shown in Equation 3, where $n$ is the population for each state.

$$
\frac{d}{d t}\left[\begin{array}{c}
n_{S 0} \\
n_{S 1} \\
n_{D}
\end{array}\right]=\left[\begin{array}{ccc}
-k_{E x} & k_{\text {rad }}+k_{\text {non-rad }} & k_{G S R} \\
k_{E x} & -\left(k_{\text {rad }}+k_{n o n-r a d}+k_{D S C}\right) & 0 \\
0 & k_{D S C} & -k_{G S R}
\end{array}\right]\left[\begin{array}{c}
n_{S 0} \\
n_{S 1} \\
n_{D}
\end{array}\right]
$$

The analytical solutions can be obtained by solving the eigenvalues and eigenvectors for rate equations (Supplementary Information S6). The observed fluorescence is proportional to the population in $\mathrm{S}_{0}$ for an ensemble measurement. The resolution of the single molecule measurement in the time domain is limited by the camera acquisition time and is significantly larger than the $\tau_{\mathrm{DSC}}$, but smaller than the GSR time constant. Therefore, our single molecule measurements provide an accurate estimation of the $k_{G S R}$, but not the $k_{D S C}$. However, we can utilize the $k_{G S R}$ values from the single molecule measurements to fit the reversible bleaching curves of the ensemble measurements to extract an accurate estimation for the $k_{D S C}$ across an irradiance range. The fluorescence decay data was fit to the analytical expression of $n_{S 0}$ to get $k_{D S C}$ values (bound $k_{G S R}$ ). The fitting results and the estimated $k_{D S C}$ values are provided in Figure 5 and Table 3. While there seems to be minimal light-driven behavior for $k_{D S C}$ for FusionRed, fitting revealed an uptick of the $k_{D S C}$ with increasing excitation rates for FusionRed-MQ.

\section{A) FusionRed}

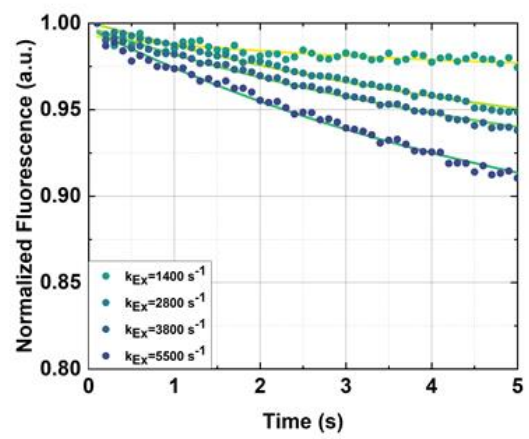

B) FusionRed-MQ

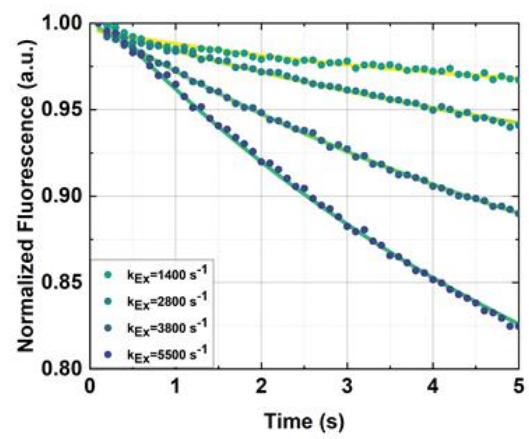

C)

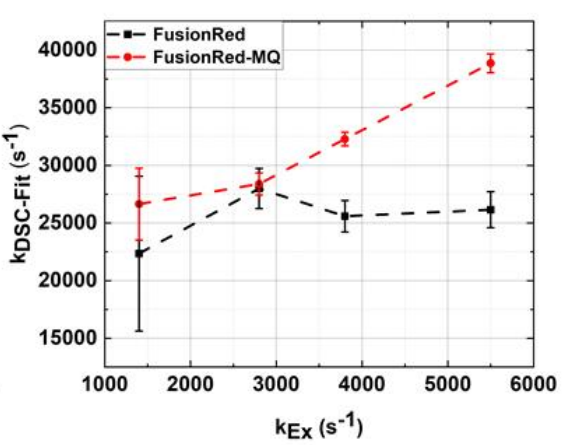

Figure 5: Photobleaching fits from a three-state model for (a) FusionRed and (b) FusionRed-MQ, across varying irradiance ranges. (c) $\mathrm{k}_{\mathrm{DSC}}$ obtained from fits. Details of the fit are provided in Supplementary Information Section S6. 
Table 3: Estimated $\mathrm{k}_{\mathrm{DSC}}$ values from fitting ensemble traces

\begin{tabular}{|c|c|c|}
\hline \multirow{2}{*}{ Excitation Rate $\left.\mathbf{s}^{-1}\right)$} & \multicolumn{2}{|c|}{ KDSC $^{\mathbf{1}}\left(\mathbf{x 1 0}^{\mathbf{3}} \mathbf{s}^{-\mathbf{1}}\right)$} \\
\cline { 2 - 3 } & FusionRed & FusionRed-MQ \\
\hline $\mathbf{1 4 0 0}$ & $22.3 \pm 6.7$ & $26.6 \pm 3.1$ \\
\hline $\mathbf{2 8 0 0}$ & $27.9 \pm 1.8$ & $28.4 \pm 0.9$ \\
\hline $\mathbf{3 8 0 0}$ & $25.6 \pm 1.4$ & $32.3 \pm 0.6$ \\
\hline $\mathbf{5 5 0 0}$ & $26.1 \pm 1.6$ & $38.9 \pm 0.8$ \\
\hline
\end{tabular}

\section{Discussion:}

Conventional models to characterize stochastic blinking usually rely on a two-state "on" and "off" system, ${ }^{28,52}$ when the excitation rate is much higher than the sampling rate of the detector used. ${ }^{65,66}$ When modeling single molecule blinking with such models under these conditions, one can assume emission takes place when the emitter is in the fluorescent $\left(\mathrm{S}_{0}\right)$ state for two consecutive simulation time steps $(<<$ dark state lifetime). Imaging methods such as widefield and TIRF $\left(\sim 10^{2-6}\right.$ photons/s) employ low excitation rates for SMLM schemes. In such cases, excitation and fluorescence photons are infrequent and long photon acquisition times are required to quantify blinking of FPs. Therefore, the detector is blind to the molecule entering or exiting the $S_{1}$ state, and needs to be modeled differently. To address this situation, we devised an algorithm incorporating Monte Carlo methods with acquisition time steps longer than excited-state lifetime but shorter than GSR time ( $100 \mathrm{~ms})$. This algorithm accurately represents our single molecule experiments, where excitation rates and acquisition timescales are comparable. With this simulation algorithm, it is reasonable to assume that one always observes the FP in $\mathrm{S}_{0}$ or $\mathrm{D}$, and $\mathrm{S}_{1}$ is only rarely populated in our three-state model. The fluorophore starts from $S_{0}$ or $D$ and returns to these states in each time cycle, and the probability of a state change in each cycle is determined by rate constants of radiative emission, internal conversion, DSC and GSR processes. Our simulation of single molecule fluorescence correctly predicted the $\tau_{\mathrm{ON}}$ dependence on $\mathrm{k}_{\mathrm{Ex}}$ and $\mathrm{k}_{\mathrm{DSC}}$, and the $\tau_{\mathrm{OFF}}$ dependence on $\mathrm{k}_{\mathrm{GSR}}$. By repeating multiple cycles of single molecule simulations, the average on and $\tau_{\mathrm{OFF}}$ can be obtained based on a probability density function of an exponential distribution: $f(t)=\frac{1}{\mu} e^{-t / \mu}$ (Supplementary Information Section S5a-e). The model was then extended to mimic ensemble behavior (Supplementary Information Section S5f-g). When varying one rate constant at a time, the ensemble dark fraction increases with increasing $\mathrm{k}_{\mathrm{Ex}}$ and $\mathrm{k}_{\mathrm{DSC}}$, but the fluorescence decay profile shows minor variation in dark fraction even with $30-45 \%$ variation in $\mathrm{k}_{\mathrm{GSR}}$. In other words, the dark fraction is controlled by factors affecting processes that populate the dark state, and the rate-determining ground state recovery process dominates the time constant of the faster component of decay in the fluorescence bleaching profile. Thus, simulations in both single molecule and ensemble average of RFPs qualitatively validated this three-state model.

We then developed a new method combining single molecule imaging and ensemble photobleaching measurements of RFPs with low irradiances to quantitatively extract dark state kinetic parameters based on the three-state model. The lowest irradiance in our single molecule measurements corresponds to an excitation rate of $\sim 1000$ photons/s. At such low irradiances, we expect an emission of less than 100 photons per frame from the dimmer FusionRed (Supplementary Information Section S7). The relatively long $\tau_{\mathrm{ON}}$ and low intensity-change thresholds for on to off (or vice versa) state changes pose a challenge for data analysis under these conditions. For these low irradiances, conventional algorithms to binarize intensity 
traces inaccurately binarize on and off events due to the low signal to noise ratios. Thresholding a change of state, in particular is a challenging step. ${ }^{61,67}$ Instead, we used algorithms based on statistics of intensity variation across frames to predict possible state change points and determine if a "real" on to off (or vice versa) event takes place. ${ }^{67}$ The analysis algorithm is also helpful to predict very short $\tau_{\mathrm{ON}}$ for the other extreme case where we use $\sim 10$-fold higher excitation rates. To accurately determine on and off events, our algorithm screened out instances where two molecules might fluoresce at the same time or the molecule undergoes permanent photobleaching (extracts $\tau_{\mathrm{OFF}}$ only when the event is bounded by an on time). The rate constants $\mathrm{k}_{\mathrm{DSC}}$ and $\mathrm{k}_{\mathrm{GSR}}$ were obtained from $\tau_{\mathrm{ON}}$ and $\tau_{\mathrm{OFF}}$ histograms and had minimal variation in the irradiance regimes examined for both FPs. The value of $\mathrm{k}_{\mathrm{GSR}}$ can be accurately determined from the single molecule fluorescence dynamics because the time resolution of the measurement, which is limited by the camera acquisition time, is smaller than the $\tau_{\mathrm{GSR}}$. Therefore, we conclusively determined that the $\mathrm{k}_{\mathrm{GSR}}$ for FusionRed is 1.3-fold higher than that of FusionRed-MQ. We also verified our hypothesis that $\mathrm{k}_{\mathrm{GSR}}$ of both FPs is independent of $\mathrm{k}_{\mathrm{Ex}}$ under low irradiances. The $\mathrm{k}_{\mathrm{DSC}}$ values are, however, on the order of $\mathrm{kHz}$, which is $\sim 2$ orders of magnitude larger than the acquisition rate $(\mathrm{Hz})$ of our single-molecule experiment. While the $\mathrm{k}_{\mathrm{DSC}}$ values are in the range of expected values from previous measurements, ${ }^{68}$ and despite high precision, accurate values of $\mathrm{k}_{\mathrm{DSC}}$ under low irradiances had to be determined by a different method.

We used an eigenvector-eigenvalue approach to extract analytical expressions for fluorescence decay from the kinetics of our three-state model and fit ensemble biexponential bleaching traces to accurately estimate the $\mathrm{k}_{\mathrm{DSC}}$. The fitting analysis revealed a light-driven dependency for the $\mathrm{k}_{\mathrm{DSC}}\left(\sim 1.5\right.$-fold increase in the $\mathrm{k}_{\mathrm{DSC}}$ with $\sim 4$-fold increase in the $\mathrm{k}_{\mathrm{ex}}$ ) of FusionRed-MQ, whereas $\mathrm{k}_{\mathrm{DSC}}$ of FusionRed remained almost constant with increasing excitation rate. While a degree of heterogeneity can be expected between single-molecule measurements obtained from pure proteins and ensemble measurements in bacterial cytoplasm, it is encouraging to note that FusionRed has low $\mathrm{pH}$ sensitivity in the neutral $\mathrm{pH}$ range and the $\mathrm{pH}$ values of our imaging buffer and bacterial cytoplasm are close $\left(\sim 7.4\right.$ vs $\sim 7.2-7.8$ for E. Coli) ${ }^{69}$ Thus, we extracted quantitative estimates for the $\mathrm{k}_{\mathrm{DSC}}$ and $\mathrm{k}_{\mathrm{GSR}}$ by combining the strengths of each approach.

Next, we consider these photophysics in the context of protein structure. The crystal structure shows that Met residue at position 42 in FusionRed is located at the imidazolinone end of the chromophore In FusionRed-MQ position 42 is substituted for a Gln residue, possibly altering the hydrogen bonding patterns at the acylimine end of the chromophore. ${ }^{53}$ Meanwhile, the residue at position 175 located above the phenol ring of the chromophore pocket; is a Leu in FusionRed and is substituted to the larger sidechain Met in FusionRed-MQ. ${ }^{53,56,70}$ The effects of these two mutations on FusionRed's brightness are complementary. Unlike the C159V mutation, Q42M and L175M do not change the profile of the ensemble bleaching, likely preserving the efficient cis to trans dark-state isomerization pathway ${ }^{53}$ It is interesting to note that the amino acid residue analogous to position 175 in FusionRed influences the dark-state behavior of the two photoconvertible FPs IrisFP and mEos4B (S173 vs F173). ${ }^{38}$ De-Zitter and co-workers demonstrated that F173 in mEos4B reduced the number of hydrogen bonds maintained by the dark chromophore in its green form compared to the smaller-sized but hydrogen-bonding capable S173 residue for IrisFP, providing an explanation for the reduced photoswitching contrast. ${ }^{38}$ As such, the $\mathrm{k}_{\mathrm{GSR}}$ values can help to identify a possible difference in barrier for dark to fluorescent state interconversion (Figure 6) between FusionRed and FusionRed-MQ. The approximate difference in the ground state barrier of bond rotation expected for a trans to cis isomerization can be calculated using a transition state theory approach for interconverting ground state isomers. ${ }^{71}$ Under the assumption that the local temperature does not change on irradiation and a similar value of the pre-exponential factor, this difference in barrier $\left(\Delta \Delta \mathrm{G}^{\#}\right)$ is $\sim 20 \mathrm{~kJ} / \mathrm{mol}$ for FusionRed 
and FusionRed-MQ. A value of $20 \mathrm{~kJ} / \mathrm{mol}$ is a reasonable free energy difference that predicts a change of a few possible hydrogen bonds, similar to what was observed for IrisFP, mEos4B and others. ${ }^{38,} 72-75$ An energetic reluctance to switch back to the bright state can therefore be explained by a conformationally restricted chromophore for FusionRed-MQ, suggesting a mechanism for the higher brightness observed for the FusionRed-L175M variant. ${ }^{53,70}$

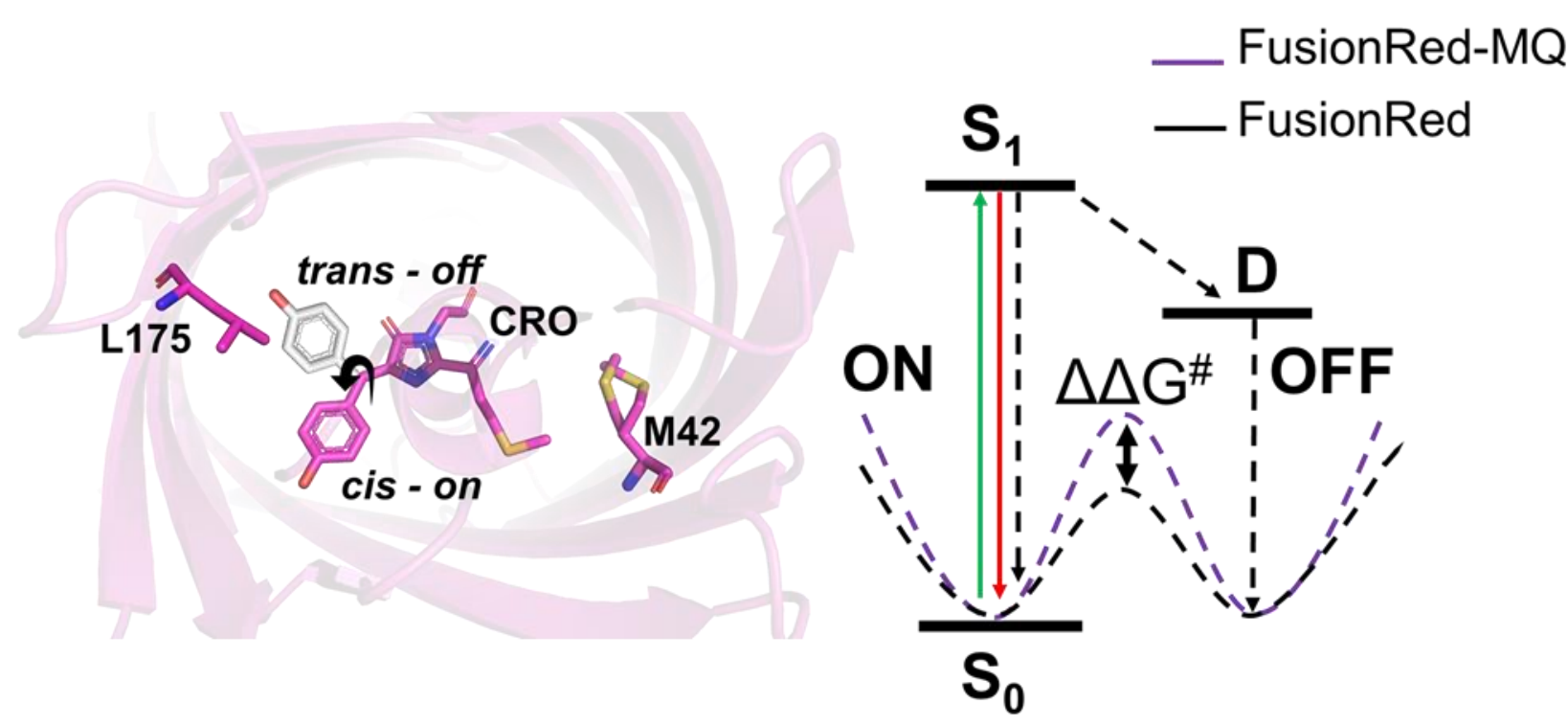

Figure 6: (A) The spatial orientation of the L175 and M42 residues in the FusionRed crystal structure (PDB ID: 6U1A), with the cis and the trans forms of the chromophore. (B) Schematic depiction of a possible barrier for a ground state cis-trans isomerization depicting a switch from an off to an on state.

\section{Conclusions}

Bright RFPs with desirable biological properties have a growing role as strong candidates for dual-usage both for SMLM-based imaging and widefield ensemble assays. ${ }^{51}$ To address the latter, in this study we quantified the nature and timescales of dark state conversion and ground state recovery for FusionRed and its 2.5-fold brighter progeny FusionRed-MQ. Our approach combined single molecule imaging with ensemble bleaching measurements to extract $\mathrm{k}_{\mathrm{DSC}}$ and $\mathrm{k}_{\mathrm{GSR}}$ of these RFPs. The methods applied in this work are an advancement over our and other previous studies where rate constants were extracted using time and frequency domain measurements at several orders of magnitude higher irradiances, which are often not suitable for widefield fluorescence and SMLM-based imaging assays ${ }^{68}$ To the best of our knowledge, this is the first study to quantify $\mathrm{k}_{\mathrm{DSC}}$ and $\mathrm{k}_{\mathrm{GSR}}$ directly under low irradiances relevant to modern SMLM imaging schemes like SOFI. We verified our hypothesis that $\mathrm{k}_{\mathrm{GSR}}$ of FusionRed and FusionRed-MQ is independent of $\mathrm{k}_{\mathrm{Ex}}$ under low irradiances. This is contrary to high irradiance studies where $\mathrm{k}_{\mathrm{GSR}}$ was observed to vary with $\mathrm{k}_{\mathrm{Ex} \cdot}{ }^{28,52}$ FusionRed is a plausible candidate for SMLM imaging, ${ }^{55}$ and our investigation suggests FusionRed-MQ is a better choice for such applications owing to its 1.8-fold higher quantum yield and higher $\mathrm{k}_{\mathrm{DSC}}{ }^{53}$ The methods and results of this work can be extended to the characterization of other fluorophores with appropriate dark state kinetic models, or incorporated into multi-parametric screening technologies to select FPs with high rates of blinking for methods like SOFI. 


\section{SUPPORTING MATERIAL}

Supplementary Information (Sections S1 to S7) have been provided to support certain results and techniques used in the manuscript. Spot Identification, trajectory binarization and single molecule analysis codes are available on GitHub: https://github.com/srijit2207/FR_DarkState.git.

\section{Corresponding Authors:}

Sheng-Ting Hung; sth@gapp.nthu.edu.tw

Ralph Jimenez; rjimenez@jila.colorado.edu

\section{Author Contributions}

S.T.H., S.M. and R.J. conceptualized the study. S.M., S.T.H. and E.S. designed and performed the experiments. S.M., C.T., S.T.H, and R.W., developed data analysis methods in the study. S.M., R.J., S.T.H. and C.T. wrote the manuscript.

\section{Notes}

The authors declare no competing financial interest.

\section{Acknowledgements}

S.M. was supported by the NIH/CU Molecular Biophysics Training Program (T32). This work was supported by the NSF Physics Frontier Center at JILA (PHY 1734006 to R.J.). C.T. thanks financial assistance from the Undergraduate Research Opportunities Program (UROP) and the Biological Science Initiative (BSI) at CU Boulder. S.T.H. is an ISAC Innovator. E.S. thanks the Astronaut Scholarship Foundation (ASF) for financial support. R.J. is a staff member in the Quantum Physics Division of the National Institute of Standards and Technology (NIST). Certain commercial equipment, instruments, or materials are identified in this paper in order to specify the experimental procedure adequately. Such identification is not intended to imply recommendation or endorsement by the NIST, nor is it intended to imply that the materials or equipment identified are necessarily the best available for the purpose. We thank Dr. Joe Dragavon, Director of the BioFrontiers Institute Advanced Light Microscopy Core facility for his valuable inputs and kind gift of fluorescent beads. We also thank Dr. Nancy Douglas, Prof. Amy Palmer, Dr. Erwin Peterman, Dr. Samantha Allen and Dr. Premashis Manna for valuable professional inputs. 


\section{References:}

1. A. D. Elliott, Confocal Microscopy: Principles and Modern Practices, Curr. Protoc. Cytom., 2020, 92, e68.

2. A. Ahmed, J. Schoberer, E. Cooke and S. W. Botchway, in Methods in Molecular Biology, ed. A. Poterszman, Springer US, New York, NY, 2021, vol. 2247, pp. 287-301.

3. H. M. Schouw, L. A. Huisman, Y. F. Janssen, R. H. J. A. Slart, R. J. H. Borra, A. T. M. Willemsen, A. H. Brouwers, J. M. van Dijl, R. A. Dierckx, G. M. van Dam, W. Szymanski, H. H. Boersma and S. Kruijff, Targeted optical fluorescence imaging: a meta-narrative review and future perspectives, Eur. J. Nucl. Med. Mol. Imaging, 2021, DOI:10.1007/s00259-021-05504-y.

4. R. Datta, T. M. Heaster, J. T. Sharick, A. A. Gillette and M. C. Skala, Fluorescence lifetime imaging microscopy: fundamentals and advances in instrumentation, analysis, and applications, J. Biomed. Opt., 2020, $25,1$.

5. W. R. Algar, N. Hildebrandt, S. S. Vogel and I. L. Medintz, FRET as a biomolecular research tool understanding its potential while avoiding pitfalls, Nat. Methods, 2019, 16, 815-829.

6. S. Duwé and P. Dedecker, Optimizing the Fluorescent Protein Toolbox and Its Use, Curr. Opin. Biotechnol., 2019, 58, 183-191.

7. G. Breuer, A formal representation of abbe's theory of microscopic image formation, Opt. Acta (Lond)., 1984, 31, 661-670.

8. J. Chojnacki and C. Eggeling, Super-resolution fluorescence microscopy studies of human immunodeficiency virus., Retrovirology, 2018, 15, 41.

9. J. Vangindertael, R. Camacho, W. Sempels, H. Mizuno, P. Dedecker and K. P. F. Janssen, An introduction to optical super-resolution microscopy for the adventurous biologist, Methods Appl. Fluoresc., 2018, 6, 22003.

10. A. S. Mishin and K. A. Lukyanov, Live-Cell Super-resolution Fluorescence Microscopy, Biochem., 2019, 84, 19-31.

11. L. Schermelleh, A. Ferrand, T. Huser, C. Eggeling, M. Sauer, O. Biehlmaier and G. P. C. Drummen, Superresolution microscopy demystified, Nat. Cell Biol., 2019, 21, 72-84.

12. L. Chen, X. Chen, X. Yang, C. He, M. Wang, P. Xi and J. Gao, Advances of super-resolution fluorescence polarization microscopy and its applications in life sciences, Comput. Struct. Biotechnol. J., 2020, 18, 2209-2216.

13. V. Schubert, P. Neumann, A. Marques, S. Heckmann, J. Macas, A. Pedrosa-Harand, I. Schubert, T.-S. Jang and A. Houben, Super-Resolution Microscopy Reveals Diversity of Plant Centromere Architecture., Int. J. Mol. Sci., , DOI:10.3390/ijms21103488.

14. S. W. Hell and J. Wichmann, Breaking the diffraction resolution limit by stimulated emission: stimulatedemission-depletion fluorescence microscopy, Opt. Lett., 1994, 19, 780.

15. E. Betzig, G. H. Patterson, R. Sougrat, O. W. Lindwasser, S. Olenych, J. S. Bonifacino, M. W. Davidson, J. Lippincott-Schwartz and H. F. Hess, Imaging intracellular fluorescent proteins at nanometer resolution, Science (80-.)., 2006, 313, 1642-1645.

16. W. E. Moerner, Microscopy beyond the diffraction limit using actively controlled single molecules, $J$. Microsc., 2012, 246, 213-220. 
17. L. Möckl and W. E. Moerner, Super-resolution Microscopy with Single Molecules in Biology and BeyondEssentials, Current Trends, and Future Challenges, J. Am. Chem. Soc., 2020, 142, 17828-17844.

18. T. Dertinger, R. Colyera, G. Iyer, S. Weiss and J. Enderlein, Fast, background-free, 3D super-resolution optical fluctuation imaging (SOFI), Proc. Natl. Acad. Sci. U. S. A., 2009, 106, 22287-22292.

19. P. D. Simonson, E. Rothenberg and P. R. Selvin, Single-molecule-based super-resolution images in the presence of multiple fluorophores, Nano Lett., 2011, 11, 5090-5096.

20. D. T. Burnette, P. Sengupta, Y. Dai, J. Lippincott-Schwartz and B. Kachar, Bleaching/blinking assisted localization microscopy for superresolution imaging using standard fluorescent molecules, Proc. Natl. Acad. Sci. U. S. A., 2011, 108, 21081-21086.

21. P. D. Dahlberg, S. Saurabh, A. M. Sartor, J. Wang, J. Wang, P. G. Mitchell, W. Chiu, W. Chiu, L. Shapiro and W. E. Moerner, Cryogenic single-molecule fluorescence annotations for electron tomography reveal in situ organization of key proteins in Caulobacter, Proc. Natl. Acad. Sci. U. S. A., 2020, 117, 13937-13944.

22. M. Lelek, M. T. Gyparaki, G. Beliu, F. Schueder, J. Griffié, S. Manley, R. Jungmann, M. Sauer, M. Lakadamyali and C. Zimmer, Single-molecule localization microscopy, Nat. Rev. Methods Prim., 2021, 1, 39.

23. Vandenberg, W.; Leutenegger, M.; Duwé, S.; Dedecker, P. An Extended Quantitative Model for SuperResolution Optical Fluctuation Imaging (SOFI). Opt. $\quad$ Express 2019, $27 \quad$ (18), 25749. https://doi.org/10.1364/oe.27.025749.

24. R. van den Eynde, A. Sandmeyer, W. Vandenberg, S. Duwé, W. Hübner, T. Huser, P. Dedecker and M. Müller, Quantitative comparison of camera technologies for cost-effective super-resolution optical fluctuation imaging (SOFI), JPhys Photonics, 2019, 1, 44001.

25 B. Moeyaert, W. Vandenberg and P. Dedecker, SOFIevaluator: a strategy for the quantitative quality assessment of SOFI data, Biomed. Opt. Express, 2020, 11, 636.

26. R. M. Dickson, A. B. Cubitt, R. Y. Tsient and W. E. Moerner, On/off blinking and switching behaviour of single molecules of green fluorescent protein, Nature, 1997, 388, 355-358.

27. M. F. Garcia-Parajo, G. M. J. Segers-Nolten, J. A. Veerman, J. Greve and N. F. Van Hulst, Real-time lightdriven dynamics of the fluorescence emission in single green fluorescent protein molecules, Proc. Natl. Acad. Sci. U. S. A., 2000, 97, 7237-7242.

28. P. Schwille, S. Kummer, A. A. Heikal, W. E. Moerner and W. W. Webb, Fluorescence correlation spectroscopy reveals fast optical excitation-driven intramolecular dynamics of yellow fluorescent proteins, Proc. Natl. Acad. Sci. U. S. A., 2000, 97, 151-156.

29. F. Malvezzi-Campeggi, M. Jahnz, K. G. Heinze, P. Dittrich and P. Schwille, Light-induced flickering of DsRed provides evidence for distinct and interconvertible fluorescent states, Biophys. J., 2001, 81, 1776-1785.

30. M. F. Garcia-Parajo, M. Koopman, E. M. H. P. Van Dijk, V. Subramaniam and N. F. Van Hulst, The nature of fluorescence emission in the red fluorescent protein DsRed, revealed by single-molecule detection, Proc. Natl. Acad. Sci. U. S. A., 2001, 98, 14392-14397.

31. U. Haupts, S. Maiti, P. Schwille and W. W. Webb, Dynamics of fluorescence fluctuations in green fluorescent protein observed by fluorescence correlation spectroscopy, Proc. Natl. Acad. Sci. U. S. A., 1998, 95, 13573-13578.

32. Heikal, A. A.; Hess, S. T.; Baird, G. S.; Tsien, R. Y.; Webb, W. W. Molecular Spectroscopy and Dynamics of Intrinsically Fluorescent Proteins: Coral Red (DsRed) and Yellow (Citrine). Proc. Natl. Acad. Sci. U. S. A. 2000, 97 (22), 11996-12001. https://doi.org/10.1073/pnas.97.22.11996. 
33. D. Sinnecker, P. Voigt, N. Hellwig and M. Schaefer, Reversible photobleaching of enhanced green fluorescent proteins, Biochemistry, 2005, 44, 7085-7097.

34. R. Berardozzi, V. Adam, A. Martins and D. Bourgeois, Arginine 66 Controls Dark-State Formation in Greento-Red Photoconvertible Fluorescent Proteins, J. Am. Chem. Soc., 2016, 138, 558-565.

35. D. Thédié, R. Berardozzi, V. Adam and D. Bourgeois, Photoswitching of Green mEos2 by Intense $561 \mathrm{~nm}$ Light Perturbs Efficient Green-to-Red Photoconversion in Localization Microscopy, J. Phys. Chem. Lett., 2017, 8, 4424-4430.

36. E. De Zitter, D. Thédié, V. Mönkemöller, S. Hugelier, J. Beaudouin, V. Adam, M. Byrdin, L. Van Meervelt, P. Dedecker and D. Bourgeois, Mechanistic investigation of $\mathrm{mEos} 4 \mathrm{~b}$ reveals a strategy to reduce track interruptions in sptPALM, Nat. Methods, 2019, 16, 707-710.

37. T. D. Krueger, L. Tang, L. Zhu, I. L. Breen, R. M. Wachter and C. Fang, Dual Illumination Enhances Transformation of an Engineered Green-to-Red Photoconvertible Fluorescent Protein, Angew. Chemie - Int. Ed., 2020, 59, 1644-1652.

38. E. De Zitter, J. Ridard, D. Thédié, V. Adam, B. Lévy, M. Byrdin, G. Gotthard, L. Van Meervelt, P. Dedecker, I. Demachy and D. Bourgeois, Mechanistic Investigations of Green mEos4b Reveal a Dynamic Long-Lived Dark State, J. Am. Chem. Soc., 2020, 142, 10978-10988.

39. A. C. Stiel, M. Andresen, H. Bock, M. Hilbert, J. Schilde, A. Schönle, C. Eggeling, A. Egner, S. W. Hell and S. Jakobs, Generation of monomeric reversibly switchable red fluorescent proteins for far-field fluorescence nanoscopy, Biophys. J., 2008, 95, 2989-2997.

40. R. Ando, H. Mizuno and A. Miyawaki, Regulated fast nucleocytoplasmic shuttling observed by reversible protein highlighting, Science (80-.)., 2004, 306, 1370-1373.

41. T. Grotjohann, I. Testa, M. Leutenegger, H. Bock, N. T. Urban, F. Lavoie-Cardinal, K. I. Willig, C. Eggeling, S. Jakobs and S. W. Hell, Diffraction-unlimited all-optical imaging and writing with a photochromic GFP, Nature, 2011, 478, 204-208.

42. G. Donnert, C. Eggeling and S. W. Hell, Major signal increase in fluorescence microscopy through darkstate relaxation, Nat. Methods, 2007, 4, 81-86.

43. D. P. Mahoney, E. A. Owens, C. Fan, J. C. Hsiang, M. M. Henary and R. M. Dickson, Tailoring Cyanine Dark States for Improved Optically Modulated Fluorescence Recovery, J. Phys. Chem. B, 2015, 119, 4637-4643.

44. A. E. Jablonski, J. C. Hsiang, P. Bagchi, N. Hull, C. I. Richards, C. J. Fahrni and R. M. Dickson, Signal discrimination between fluorescent proteins in live cells by long-wavelength optical modulation, J. Phys. Chem. Lett., $2012,3,3585-3591$.

45. Y. C. Chen, A. E. Jablonski, I. Issaeva, D. Bourassa, J. C. Hsiang, C. J. Fahrni and R. M. Dickson, Optically Modulated Photoswitchable Fluorescent Proteins Yield Improved Biological Imaging Sensitivity, J. Am. Chem. Soc., 2015, 137, 12764-12767.

46. B. C. Fleischer, J. T. Petty, J. C. Hsiang and R. M. Dickson, Optically Activated Delayed Fluorescence, J. Phys. Chem. Lett., 2017, 8, 3536-3543.

47. D. P. Mahoney, A. A. Demissie and R. M. Dickson, Optically Activated Delayed Fluorescence through Control of Cyanine Dye Photophysics, J. Phys. Chem. A, 2019, 123, 3599-3606.

48. B. Peng, R. Dikdan, S. E. Hill, A. C. Patterson-Orazem, R. L. Lieberman, C. J. Fahrni and R. M. Dickson, Optically Modulated and Optically Activated Delayed Fluorescent Proteins through Dark State Engineering, J. Phys. Chem. B, 2021, 125, 5200-5209. 
49. S. Waldchen, J. Lehmann, T. Klein, S. Van De Linde and M. Sauer, Light-induced cell damage in live-cell super-resolution microscopy, Sci. Rep., 2015, 5, 15348.

50. P. P. Laissue, R. A. Alghamdi, P. Tomancak, E. G. Reynaud and H. Shroff, Assessing phototoxicity in live fluorescence imaging, Nat. Methods, 2017, 14, 657-661.

51. S. Mukherjee and R. Jimenez. Photophysical Engineering of Fluorescent Proteins: Accomplishments and Challenges of Physical Chemistry Strategies. J. Phys. Chem B. 2022, (Accepted)

52. A. Schenk, S. Ivanchenko, C. Röcker, J. Wiedenmann and G. U. Nienhaus, Photodynamics of Red Fluorescent Proteins Studied by Fluorescence Correlation Spectroscopy, Biophys. J., 2004, 86, 384-394.

53. S. Mukherjee, S. T. Hung, N. Douglas, P. Manna, C. Thomas, A. Ekrem, A. E. Palmer and R. Jimenez, Engineering of a brighter variant of the FusionRed fluorescent protein using lifetime flow cytometry and structureguided mutations, Biochemistry, 2020, 59, 3669-3682.

54. W. Weber, V. Helm, J. A. Mccammon and P. W. Langhoff, Shedding light on the dark and weakly fluorescent states of green fluorescent proteins, Proc. Natl. Acad. Sci. U. S. A., 1999, 96, 6177-6182.

55. N. V. Klementieva, A. I. Pavlikov, A. A. Moiseev, N. G. Bozhanova, N. M. Mishina, S. A. Lukyanov, E. V. Zagaynova, K. A. Lukyanov and A. S. Mishin, Intrinsic blinking of red fluorescent proteins for super-resolution microscopy, Chem. Commun., 2017, 53, 949-951.

56. L. Muslinkina, V. Z. Pletnev, N. V. Pletneva, D. A. Ruchkin, D. V. Kolesov, A. M. Bogdanov, L. A. Kost, T. V. Rakitina, Y. K. Agapova, I. I. Shemyakina, D. M. Chudakov and S. Pletnev, Two independent routes of posttranslational chemistry in fluorescent protein FusionRed, Int. J. Biol. Macromol., 2020, 155, 551-559.

57. F. Pennacchietti, E. O. Serebrovskaya, A. R. Faro, I. I. Shemyakina, N. G. Bozhanova, A. A. Kotlobay, N. G. Gurskaya, A. Bodén, J. Dreier, D. M. Chudakov, K. A. Lukyanov, V. V. Verkhusha, A. S. Mishin and I. Testa, Fast reversibly photoswitching red fluorescent proteins for live-cell RESOLFT nanoscopy, Nat. Methods, 2018, 15, 601-604.

58. M. Honda, Y. Saito, N. I. Smith, K. Fujita and S. Kawata, Nanoscale heating of laser irradiated single gold nanoparticles in liquid, Opt. Express, 2011, 19, 12375.

59. J. L. Davis, B. Dong, C. Sun and H. F. Zhang, Method to identify and minimize artifacts induced by fluorescent impurities in single-molecule localization microscopy, J. Biomed. Opt., 2018, $23,1$.

60. C. Tregidgo, J. A. Levitt and K. Suhling, Effect of refractive index on the fluorescence lifetime of green fluorescent protein, J. Biomed. Opt., 2008, 13, 031218.

61. L. P. Watkins and H. Yang, Detection of intensity change points in time-resolved single-molecule measurements, J. Phys. Chem. B, 2005, 109, 617-628.

62. H. F. Ng, Automatic thresholding for defect detection, Pattern Recognit. Lett., 2006, 27, $1644-1649$.

63. L. Kamentsky, T. R. Jones, A. Fraser, M. A. Bray, D. J. Logan, K. L. Madden, V. Ljosa, C. Rueden, K. W. Eliceiri and A. E. Carpenter, Improved structure, function and compatibility for CellProfiler: Modular high-throughput image analysis software, Bioinformatics, 2011, 27, 1179-1180.

64. W. T. Yip, D. Hu, J. Yu, D. A. Vanden Bout and P. F. Barbara, Classifying the photophysical dynamics of single- and multiple-chromophoric molecules by single molecule spectroscopy, J. Phys. Chem. A, 1998, 102, 75647575 . 
65. E. J. G. Peterman, S. Brasselet and W. E. Moerner, The fluorescence dynamics of single molecules of green fluorescent protein, J. Phys. Chem. A, 1999, 103, 10553-10560.

66. B. Lounis, J. Deich, F. I. Rosell, S. G. Boxer and W. E. Moerner, Photophysics of DsRed, a red fluorescent protein, from the ensemble to the single-molecule level, J. Phys. Chem. B, 2002, 105, 5048-5054.

67. H. Yang, H. Li and T. Liu, Photobleaching statistics in single-molecule on-/off-time distributions, J. Chem. Phys., 2019, 151, 174101.

68. P. Manna and R. Jimenez, Time and frequency-domain measurement of ground-state recovery times in red fluorescent proteins, J. Phys. Chem. B, 2015, 119, 4944-4954.

69. J. C. Wilks and J. L. Slonczewski, pH of the cytoplasm and periplasm of Escherichia coli: Rapid measurement by green fluorescent protein fluorimetry, J. Bacteriol., 2007, 189, 5601-5607.

70. P. Manna, S. T. Hung, S. Mukherjee, P. Friis, D. M. Simpson, M. N. Lo, A. E. Palmer and R. Jimenez, Directed evolution of excited state lifetime and brightness in FusionRed using a microfluidic sorter, Integr. Biol. (United Kingdom), 2018, 10, 516-526.

71. C.-Y. Lin, M. Romei, I. Mathews and S. Boxer, Energetic basis of excited-state enzyme design and function, ChemRxiv, 2021.

72. X. He, A. F. Bell and P. J. Tonge, Ground state isomerization of a model green fluorescent protein chromophore, FEBS Lett., 2003, 549, 35-38.

73. S. Rafiq, B. K. Rajbongshi, N. N. Nair, P. Sen and G. Ramanathan, Excited state relaxation dynamics of model green fluorescent protein chromophore analogs: Evidence for cis-trans isomerism, J. Phys. Chem. A, 2011, 115, 13733-13742.

74. E. Carrascosa, J. N. Bull, M. S. Scholz, N. J. A. Coughlan, S. Olsen, U. Wille and E. J. Bieske, Reversible Photoisomerization of the Isolated Green Fluorescent Protein Chromophore, J. Phys. Chem. Lett., 2018, 9, 26472651.

75. J. Chang, M. G. Romei and S. G. Boxer, Structural Evidence of Photoisomerization Pathways in Fluorescent Proteins, J. Am. Chem. Soc., 2019, 141, 15504-15508. 